\title{
SATURATION IN HUMAN CONES
}

\author{
STEVEN K. SHEVELL \\ Department of Psychology, The University of Michigan, \\ Ann Arbor, MI 48104, U.S.A. \\ (Received 18 August 1976; in revised form 30 August 1976)
}

\begin{abstract}
Increment threshold intensities were measured for a foveally presented 20 msec test flash which fell on a flashed $4.1^{\circ}$ white background field. The test field was varied in color (red or green) and size $\left(1^{\circ}\right.$ or $\left.0.57^{\circ}\right)$. In one condition (green $0.57^{\circ}$ test) the onset asynchrony of the background and test fields was also varied. The increment threshold measurements directly demonstrate saturation of the $\pi_{4}$ and $\pi_{5}$ color mechanisms and, based on the cone signal equation given by Alpern, Rushton and Torii $(1970 \mathrm{c})$, provide estimates of the semi-saturation constant $\sigma$. For a given color mechanism, a single value of $\sigma$ was found to be adequate for all test flash sizes and asynchrony intervals tested; however, these values of $\sigma$ are about 100 times smaller than those found by Alpern et al. using the contrast-flash technique.

The Alpern et al. cone signal equation is essentially a simple gain control model. It is shown that when light intensities are correctly expressed in terms of quantum catch, the gain control model can account explicitly for both the observed cone mechanism saturation in the absence of pigment bleaching and the absence of saturation in the presence of bleaching by steady backgrounds.
\end{abstract}

Key Words-cone saturation; cone signals.

It is well known that the human cone visual system cannot be saturated by a steady background light (Stiles, 1939, 1949, 1959). For a background light of any intensity (no matter how strong), it is always possible to see a sufficiently strong test flash superimposed on the steady background. Quite the opposite is found in the rod system, in which a steady, moderately strong background (about 3 log scotopic td) raises the increment threshold so high that no test flash of any intensity can be detected (Aguilar and Stiles, 1954).

In a series of papers, Alpern, Rushton and Torii $(1970 \mathrm{a}, \mathrm{b}, \mathrm{c})$ investigated the saturation of both rods and cones. Rather than using a conventional increment threshold paradigm, Alpern et al. employed the contrast-flash technique (Alpern, 1965; Alpern and Rushton, 1965, 1967) in which the intensity of an after-flash surrounding (but not including) the test field is adjusted so as to bring the test flash to threshold. They demonstrated saturation by adding a steady background light to the surround area only and then measuring the intensity of the after-flash required to bring a fixed test to threshold as a function of steady background intensity. Alpern et al. (1970c) also suggested a modification to the standard increment threshold procedure which involved the presentation of a brief test on a flashed background. Using this technique with a white, foveally presented test falling on a flashed white background, they showed that a background of about $6 \log$ td saturates the photopic system. King-Smith and Webb (1974) used the same procedure with colored test and background fields to demonstrate saturation in each of the red, green, and blue color mechanisms. However, they provided only relative intensity measurements.

The present experiments use the same experimental paradigm. Increment threshold measurements were made for a foveally presented $20 \mathrm{msec}$ test flash (denoted $\lambda$ ) which fell on a flashed, $4.1^{\circ}$ white back- ground field (denoted $\theta$ ). In addition, conventional increment threshold measurements were made with the test field $\lambda$ falling on a steady background $\theta$ presented for a period sufficiently long to assure that the level of bleached pigment was at equilibrium.

The present increment threshold measurements made with fiashed backgrounds provide a direct demonstration of $\pi_{4}$ and $\pi_{5}$ color mechanism saturation. The effect of test field size is also explored, as is the effect of altering the test and background fields' onset asynchrony. Conventional increment threshold measurements make possible a comparison between measurements made with flashed and steady backgrounds. By measuring absolute (rather than relative) test light and background light intensities, it is possible to relate the present increment threshold measurements (1) to previously published experimental results and (2) to the amount of pigment bleached by the background lights. Absolute intensity measurements allow precise quantitative development of a gain control model in which the gain depends on the quantum catch of the incident background light. Absolute intensities are necessary because the fraction of cone pigment bleached affects the quantum catch. It is shown that this model can account explicitly for the saturation observed with flashed backgrounds and the absence of saturation found with steady backgrounds.

\section{METHOD}

\section{Procedure}

Each experimental trial was composed of a white background flash of some intensity (sometimes zero) on top of which was presented a smaller red or green test flash. The onset of the background flash preceded the onset of the test flash by the interval $\delta$, and the test flash remained on for $20 \mathrm{msec}$. The background flash remained on an additional 140 msec after the test nash went of (see Fig. 1a). 
(a)

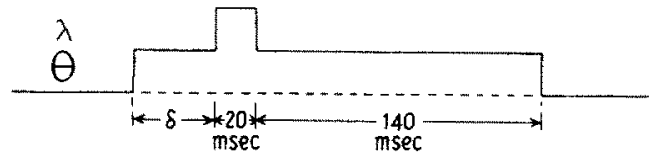

(b)

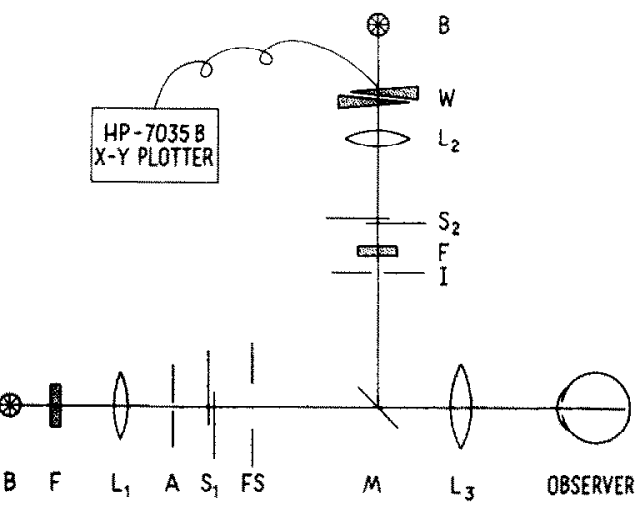

Fig. 1. (a) Representation of the test flash $\lambda$ and background field $\theta$ in time. (b) Schematic drawing of the apparatus.

The background field was always $4.1^{\circ}$, and the test field was either $1^{\circ}$ or $0.57^{\circ}$. Both fields were centered at the fovea with fixation being aided by two fixation points, one 1.5 above the other $1.5^{\circ}$ below the center of the field (the sub ject fixating midway between). The subject (SS) always adjusted the intensity of the test flash for threshold. A vertical cross hair was placed across the test field because high intensity backgrounds often resulted in test flash threshold levels which produced afterimages (see Geisler. 1975). The subject found that the cross hair eliminated confusion between the test flash and its afterimage. Detection of the cross hair was the threshold criterion in all experiments.

All threshold measurements were made on the subject's right eye. The subject wore his normal prescription lenses $\left(-1.25 \mathrm{sph}-0.25 \mathrm{cyl}\right.$. axis $\left.45^{\prime}\right)$. The presentation rate of trials was approx $1 / \mathrm{sec}$ except when $\delta$ was $250 \mathrm{msec}$, in which condition the rate was approx 1 trial every $2 \mathrm{sec}$. For sessions in which a steady background field $(\delta \neq x)$ was used, the subject adapted to the given background fot $10 \mathrm{~min}$ before beginning increment threshold measurements. The $20 \mathrm{msec}$ test flash was then presented at a rate of $1 \mathrm{ftash} / \mathrm{sec}$.

\section{Apparatus}

A diagram of the apparatus is shown in Fig. $1 \mathrm{~b}$. The design of the experiments required the presentation of two fields (a background field $\theta$ and a test field $\lambda$ ) which could be independently controlled for intensity and size. Identical but separate sources (B) were $6 \mathrm{~V}$ tungsten filament bulbs. In the background field channel, a lens $L_{1}$ (focal length $57 \mathrm{~mm}$ ) imaged the flament on the aperture stop $\mathrm{A}$ which was immediately followed by a Vincent Uniblitz electronic shutter $S_{1}$. Beyond the shutter was a fixed size $4.1^{\circ}$ field stop FS which was located exactly one focal length from the Maxwellian lens $L_{3}$, a Kodak Aero Ektar lens $U / 2.5$, focal length $155 \mathrm{~mm}$ ). The intensity of the test beam was controlled by a compensated neutral density wedge W which immediately followed the source. A lens $L_{2}$ (focal length $57 \mathrm{~mm}$ ) imaged the light in front of a second Vincent Uniblitz electronic shutter $S_{2}$ beyond which was an iris $l$, located one focal length from $L_{3}$, which served as a variable size field stop. Attached to the iris (on the surface facing lens $L_{3}$ ) were two very low intensity lights which served as fixation points, and a vertical cross hair which approximately bisected the circular test field.

The test and background fields were merged by a halfsilvered mirror $M$. The source images formed by lenses
$L_{1}$ and $L_{2}$ were in tum imaged in the plane of the subjecls pupil by lens $L_{3}$. These images were less than $2 \mathrm{~mm}$ in size. consequently smaller than the smallest possible natural pupil. Neutral density and colored filters $F$ could be placed in either beam. All of the equipment was mounted on standard optical bench saddles locked onto triangular rails which were securely fastened to a table. The entire apparatus was enclosed in black cardboard to reduce, though never completely exclude. scattered light,

The subject controlled the intensity of the lest flash by turning a knob which adjusted the wedge by means of a mechanical linkage. The shaft on which the wedge rotated was coupled to a potentiometer, so that the position of the wedge could be monitored by measuring the voltage across the potentiometer. This voltage, and thus the wedge position and therefore the test feld intensily, was recorded on a Hewlett-Packard HP-7035B X Y plotter.

\section{Calibration}

The intensity of each source was measured by allowing the beam to fall on a white diffusing surface of known reflectivity $80 \mathrm{~mm}$ beyond the image produced by lens $L_{3}$. The luminance of the surface was measured using an S.E.I. photometer, and the intensity of the source then calculated as described by Westheimer (1966). The intensity of the white (tungsten) background field source was $6.10 \mathrm{log}$ td. The intensity of the tungsten test field source was $5.66 \mathrm{log}$ $\mathrm{td}$. Using a Beckman spectrophotometer, the transmission of each Wratten filter used was measured for every $10 \mathrm{~nm}$ step hetween 400 and $700 \mathrm{~nm}$. The overall transmission for each filter was then computed by integration, assuming illuminant (A) and CIE photopic sensitivity. The maximum test field intensity was $4.84 \mathrm{log}$ td with the (red) Wratten 26 filter (transmission $15.3 \%$ ) in the beam, and $5.03 \log$ td with the (green) Wratten 57 filter (transmission $23.6 \%$ ) in place. The test flash was always presented for $20 \mathrm{msec}$. implying maximum test field intensities of 3.14 and 3.33 $\log$ td sec, respectively.

The wedge was calibrated at two wavelengths, 500 and $610 \mathrm{~nm}$. The spectral transmissivity of the Inconel wedge was sufficiently flat within a moderate wavelength range so that the spectral distribution of the source could be ignored.

At the beginning of every session the subject adjusted the position of the bite bar so that the center of his pupil was at the image of the flaments formed by lens $L_{3}$. A measurement of absolute threshold (no background) always followed immediately as an extra check on the current calibration. The day to day consistency of this measurement was sufficiently good to detect a malfunction (which occurred in the test beam source) by noting an increase in absolute threshold of $0.20 \mathrm{log} \mathrm{td}$ sec.

\section{RESULTS}

\section{$\pi_{5}$ Mechanism}

The white (tungsten) background light is broad band and therefore the thresholds for all cone mechanisms are usually raised. Stiles $(1939,1949$ $1959)$ has shown that distinct retinal mechanisms can be isolated that act independently in increment threshold measurements. King-Smith and Webb (1974) demonstrated that cone mechanism independence holds for flashed (as well as steady) backgrounds. Using a white background, it is assumed that the dominant wavelength of the test field determines which cone mechanism provides the threshold sensation. With a Wratten 26 filter (dominant wavelength $620 \mathrm{~nm}$ ) in the test beam, it is assumed that only the $\pi_{5}$ mechanism is contributing to the thresh- 


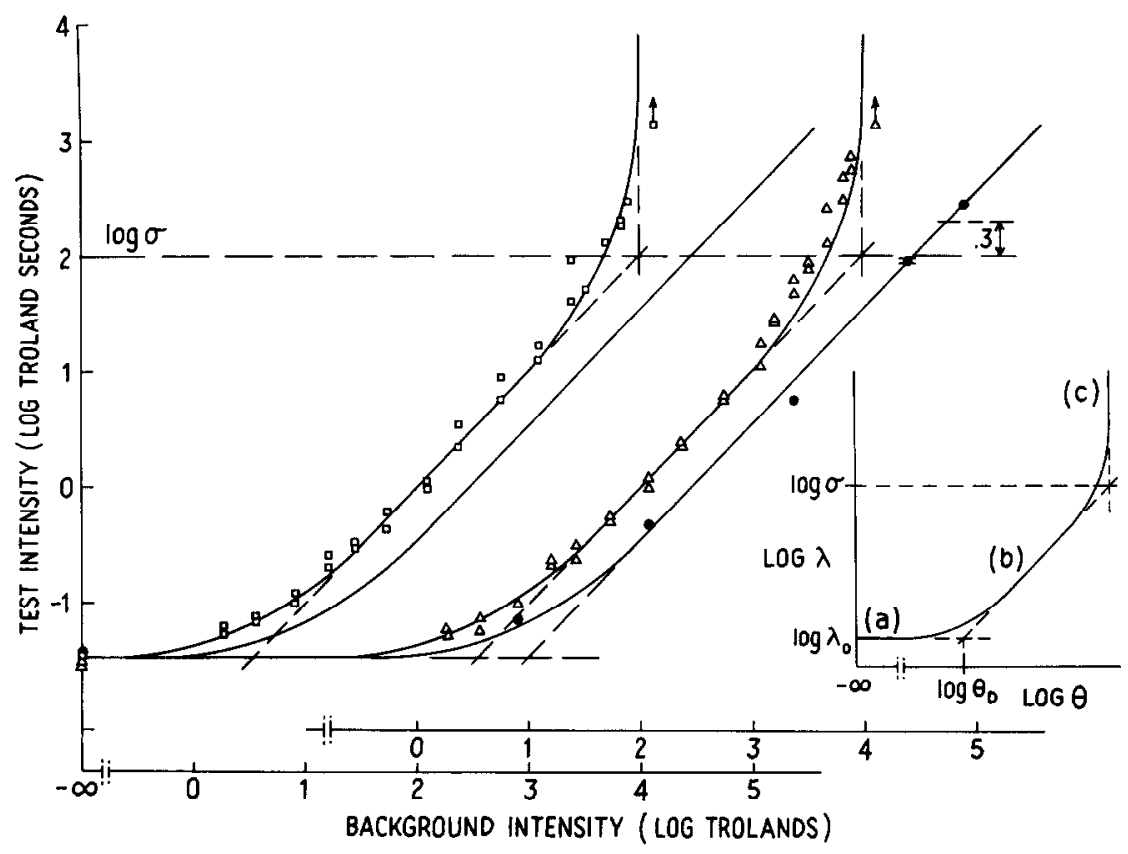

Fig. 2. Increment threshold measurements for the $\pi_{5}$ mechanism (dominant wavelength of test flash $620 \mathrm{~nm}$, Wratten 26 filter). Squares are measurements with a $1^{\circ}$ test and flashed backgrounds $(\delta=40 \mathrm{msec}$, lower abscissa); triangles are measurements with a $0.57^{\circ}$ test and flashed backgrounds $(\delta=40 \mathrm{msec}$, upper abscissa); circles are measurements with a $0.57^{\circ}$ test and steady backgrounds $(\delta=\infty$, upper abscissa). The curve through the circles is replotted on the lower abscissa for reference. The inset is a schematic plot of $\log \lambda$ vs $\log \theta$ for constant $N$ according to equation (1).

old measurements. The experiments of King-Smith and Webb (1974) give very strong support to this assumption. While in other work King-Smith (1975) has recently suggested that this may not be exactly true, as a first approximation it is unlikely to be very much in error.

Figure 2 shows increment threshold measurements for the $\pi_{5}$ mechanism. Each data point is the average of at least three measurements made in a single session. Open symbols indicate results with the asynchrony interval $\delta$ fixed at $40 \mathrm{msec}$. The triangles are measurements with a $0.57^{\circ}$ test field (upper abscissa); the squares are measurements with a $1^{\circ}$ test field (lower abscissa). The filled circles are increment threshold measurements with a $0.57^{\circ}$ test falling on a conventional steady $(\delta=\infty)$ background (upper abscissa). The solid curves drawn through the open symbols are identical up to a horizontal shift, and are according to the Alpern et al. (1970c) cone signal equation; they will be discussed later. The curve through the filled circles is according to Weber's law (after adding to each background intensity a constant factor for noise).

The data in Fig. 2 clearly demonstrate saturation of the $\pi_{5}$ mechanism when the background field is a brief flash (open symbols). The two test field sizes give virtually identical increment threshold intensities. The increment threshold measurements made with steady backgrounds show no saturation, a very well known experimental result.

\section{$\pi_{4}$ Mechanism}

In order to measure increment thresholds for the $\pi_{4}$ mechanism, the red Wratten filter in the test beam was replaced by a (green) Wratten 57 filter (dominant wavelength $530 \mathrm{~nm}$ ). With the green test flash falling on the white background it is assumed (and strongly supported by the data of King-Smith and Webb (1974)) that only the $\pi_{4}$ mechanism is contributing to the threshold measurements.

The open squares and upright triangles in Fig. 3 display $\pi_{4}$ mechanism measurements analogous to those reported above for $\pi_{5}$. The asynchrony interval $\delta$ is $40 \mathrm{msec}$; the squares represent observations with a $1^{\circ}$ test field, while the triangles indicate measurements made with a $0.57^{\circ}$ test field (middle abscissa). Each data point represents the average of at least three measurements made in a single session. The two sets of measurements virtually overlie one another, indicating no effect due to test field size.

An additional experiment with the $\pi_{4}$ mechanism investigated the effect of varying $\delta$. The above results for $\pi_{5}$ and $\pi_{4}$ indicate no significant test field size effect, so the following measurements were made with the $0.57^{\circ}$ test field only. Figure 3 shows increment threshold measurements made with $\delta=20 \mathrm{msec}$ (open inverted triangles, lower abscissa), $\delta=40 \mathrm{msec}$ (open upright triangles, middle abscissa), $\delta=250$ msec (open diamonds, upper abscissa) and $\delta=\infty$ (filled circles, upper abscissa). A single shaped curve (adjusted only horizontally), again according to Alpern et al.'s (1970c) cone signal equation, is fitted to each set of measurements made with the $0.57^{\circ}$ test field and flashed backgrounds. The curve through the measurements made with steady backgrounds, which is according to Weber's law, is redrawn on the lower and middle abscissas to show its distance from the curves for each asynchrony interval. 


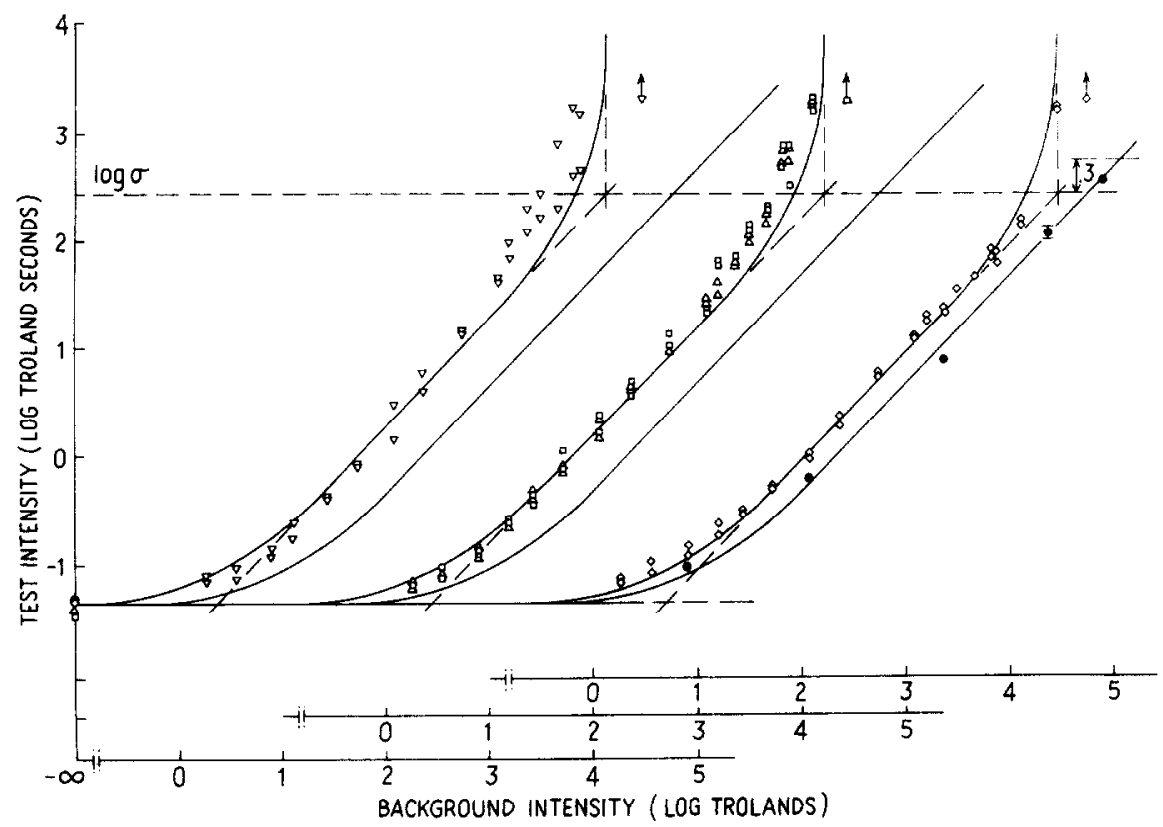

Fig. 3. Increment threshold measurements for the $\pi_{4}$ mechanism (dominant wavelength of test flash $530 \mathrm{~nm}$, Wratten 57 filter). Open symbols represent measurements with flashed backgrounds with $\delta=20 \mathrm{msec}$ and a $0.57^{\circ}$ test (inverted triangles, lower abscissa), $\delta=40 \mathrm{msec}$ and a $0.57^{\circ}$ test (upright triangles, middle abscissa), $\delta=40 \mathrm{msec}$ and a $1^{\circ}$ test (squares, middle abscissa), and $\delta=250 \mathrm{msec}$ and a $0.57^{\circ}$ test (diamonds, upper abscissa). Filled circles represent measurements with steady backgrounds and a $0.57^{\circ}$ test (upper abscissa). The curve through the circles is replotted on the lower and middle abscissas for reference.

\section{DISCUSSION}

The data clearly demonstrate saturation of the $\pi_{4}$ and $\pi_{5}$ color mechanisms when the background field is a brief flash. The two test field sizes used give virtually indistinguishable increment threshold functions. The major effect of reducing the onset asynchrony (at least in the range tested) is to shift the increment threshold function to the left; there also appears to be a slight change in the functions' shapes at the highest background intensities (Fig. 3). The above experiments also show that background intensities that saturate the color mechanisms when presented as brief flashes do not saturate the color mechanisms when presented as steady backgrounds allowed to reach bleaching equilibrium. It will be shown later that a quite simple gain control model, where intensities are expressed in terms of quantum catch in order to include the effect of pigment depletion, can account for both saturation with flashed backgrounds and the absence of saturation with steady backgrounds.

\section{Comparisons with previous results}

Alpern et al. (1970a,b,c) used the contrast-flash technique (frequently with modification) to measure psychophysically signals from rods and cones. Of specific interest here is their cone signal equation (Alpern et al., 1970c) which states that the signal due to a flash of intensity $\lambda$ falling on a background field of intensity $\theta$ is

$$
N=\frac{\lambda}{\lambda+\sigma} \frac{\theta_{D}}{\theta+\theta_{D}}
$$

where $\lambda$ and $\theta$ are expressed in units of quantum catch, $\sigma$ is the semi-saturation constant (i.e. setting $\theta=0, \sigma$ is the value of $\lambda$ for which the cone signal $N$ is one-half its maximum), and $\theta_{D}$ is a constant interpreted as sensory noise (eigengrau). In the absence of a background field (i.e. when $\theta=0$ ), $N=\lambda /(\lambda+\sigma)$. The background light attenuates this signal by the multiplicative factor $\theta_{D} /\left(\theta+\theta_{D}\right)$. In the absence of pigment bleaching, values for $\lambda$ and $\theta$ may be expressed in td (or td $\mathrm{sec}$ ), since conversion to quantum catch units only requires multiplication by a constant.

Equation (1) was derived by measuring inhibitory receptor signals from contrast-flashes, but Alpern et al. $(1970 \mathrm{c})$ have extended it to the excitatory signals of increment thresholds. According to their formulation, the incremental test flash $\lambda$ will be detected only when the signal resulting from the combined effects of the test and background fields is greater than the minimum, critical signal for threshold $N_{c^{*}}$. It is assumed that the signals in the $\pi_{4}$ and $\pi_{5}$ cone mechanisms act independently, and thus equation (1) holds separately for each mechanism. The constants $\sigma$ and $\theta_{D}$ may vary between mechanisms. Equation (1) implies an increment threshold curve of a very distinct shape and provides a simple method for estimating the model parameters $\sigma$ and $\theta_{D}$ from increment threshold data.

Consider a schematic representation of the increment threshold curve implied by equation (1) (inset, Fig. 2) in which $\log \lambda$ is plotted on the ordinate and $\log \theta$ is along the abscissa. The curve represents $\log \lambda$ as a function of $\log \theta$ for a constant $N$, since every point on the curve represents an assumed fixed level of cone signal (threshold level $N_{c}$, in all of the experiments discussed above). The 
curve contains three linear segments which are direct consequences of equation (1).

The horizontal segment (a) represents the absolute threshold measurement. For the data reported here, the absolute threshold test intensity $\lambda_{0}$ is over three orders of magnitude smaller than $\sigma$. Thus, the approximation provided by $\lambda_{0} \ll \sigma$ yields

$$
N_{t}=\frac{\lambda_{0}}{\sigma}
$$

since $\theta=0$ at absolute threshold. This must be the value of (fixed) $N_{c}$ for the entire curve.

Segment (b) represents the portion of the curve where $\theta$ is sufficiently large and $\lambda$ still sufficiently small so the approximations $\theta \gg \theta_{D}$ and $\lambda \ll \sigma$ both hold. In this case

$$
N_{c}=\frac{\lambda_{0}}{\sigma}=\frac{\lambda \theta_{D}}{\sigma} \frac{1}{\theta}
$$

which, upon taking logarithms and rearranging, gives

$$
\log \lambda=\log \theta+\log \left(\frac{\lambda_{0}}{\theta_{D}}\right)
$$

The quantity $\left(\lambda_{0} / \theta_{D}\right)$ is constant, and therefore segment (b) is a linear segment with slope 1.0 .

By substituting $\lambda=\lambda_{0}$ into equation (4), the equality may be rearranged to yield $\log \theta=\log \theta_{b}$. Thus the downward extension of the $45^{\circ}$ line, segment (b), intersects the horizontal extension of segment (a) at $\log \theta=\log \theta_{D}$ (see inset, Fig. 2).

Now consider segment (c). In this portion of the curve $\lambda$ is no longer smaller than $\sigma$, but clearly $\theta \gg \theta_{D}$. Therefore

$$
N_{c}=\frac{\lambda}{\lambda+\sigma} \frac{\theta_{D}}{\theta}
$$

At the point where saturation occurs, the background intensity $\theta$ is just large enough so that only an infinitely intense test fiash $\lambda$ can be detected. At this level of $\theta$,

$$
N_{e}=\frac{\lambda_{\theta}}{\sigma}=\frac{\theta_{D}}{\theta} \text { since } \lim _{\lambda \rightarrow \infty} \frac{\lambda}{\lambda+\sigma}=1 .
$$

Thus saturation occurs at

$$
\theta=\frac{\sigma \theta_{D}}{\lambda_{0}} .
$$

Substituting this value of $\theta$ into equation (4), the equation for segment (b), reveals $\log \lambda=\log \sigma$. Thus the upward extension of the $45^{\circ}$ line, segment (b), intersects the downward vertical extension of segment (c) at $\log \lambda=\log \sigma$ (see inset, Fig. 2). Therefore the data reported in Figs. 2 and 3 provide estimates of $\sigma$ for the $\pi_{4}$ and $\pi_{5}$ mechanisms.

The solid curves through the two sets of open symbols (fiashed backgrounds) in Fig. 2 are identical in shape and are according to equation (1). A horizontal adjustment of the curves was made by fitting the measurements falling on the $45^{\circ}$ linear segments. The data fit the curves well; the value of the semi-saturation constant $\sigma$ for the $\pi_{s}$ mechanism is about 2.0 $\log t \mathrm{sec}$. The lower portion of the curve through the filled circles (steady backgrounds) is a horizontal displacement of the lower part of the curve through the open symbols, and the upper portion is simply

${ }^{1}$ The invariance of $\sigma$ with changes in onset asynchrony does not imply that the background intensity at which cone signals saturate must also be independent of the asynchrony. The saturating intensity does indeed vary with onset asynchrony, thus indicating the presence of neural transient effects. an extension of the $45^{\circ}$ line. This curve expresses the familiar Weber-Fechner relationship since segments (a) and (b) of Fig. 2 (inset) are based on $\lambda \ll \sigma$ in which case equation (1) reduces to

$$
N_{c}=\frac{\lambda_{0}}{\sigma}=\frac{\lambda}{\sigma} \frac{\theta_{D}}{\theta+\theta_{D}} .
$$

Since $\lambda_{0}$ and $\theta_{D}$ are constant,

$$
\frac{\lambda}{\theta+\theta_{D}}=\frac{\lambda_{0}}{\theta_{D}}=\text { constant }
$$

This curve is redrawn on the lower abscissa of Fig. 2 to show its position relative to the measurements indicated by squares.

In Fig. 3 a (second) single shaped curve (adjusted only horizontally) has been fitted to each set of measurements made with the smaller test field falling on a flashed background. The curve is according to equation (1), and indicates that the semi-saturation constant $\sigma$ for the $\pi_{4}$ mechanism is about $2.45 \log$ td sec. This single shaped curve fits all of the observations well except for those points representing high intensity backgrounds presented with short $(40 \mathrm{msec}$ or less) asynchrony intervals. These points tend to rise slightly above the curve, an issue which will be considered below. The Weber's law curve through the filled circles (steady backgrounds) is redrawn on the lower and middle abscissas.

Overall, the curves implied by the Alpern et al. cone signal equation [equation (1)] fit the data well. Only horizontal adjustments of each single shaped curve were required for adequate fits to the flashed background data; thus for a given color mechanism, a single value of the semi-saturation constant $\sigma$ was sufficient for all conditions tested. ${ }^{1}$ The semi-saturation constant $\sigma$ of $2.45 \log$ td $\mathrm{sec}$ found for the $\pi_{4}$ mechanism is larger than the $2.0 \mathrm{log}$ td $\mathrm{sec}$ value found for the $\pi_{5}$ mechanism. Since it has been assumed that independent cone mechanisms are providing the threshold sensation, a comparison of the $\pi_{4}$ and $\pi_{5}$ semi-saturation constants may be more meaningful when the $\sigma$ values are expressed in units of " $\pi_{4}$ td sec" and " $\pi_{5}$ td sec", respectively. Intensities of lights in $\pi_{4}$ (or $\pi_{5}$ ) td were calculated by substituting the $\pi_{4}$ (or $\pi_{5}$ ) spectral sensitivity curve [an energy basis curve, which was computed from quantal basis data given by Wyszecki and Stiles (1967)] for the photopic sensitivity curve, and then integrating in the usual manner. This calculation shows that $\sigma$ for the $\pi_{4}$ mechanism is $2.46 \log \pi_{4}$ td sec and $\sigma$ for the $\pi_{5}$ mechanism is $2.21 \log \pi_{5}$ td sec. Converting the energy based $\pi_{4}$ and $\pi_{5}$ td units to quanta reveals that $\sigma$ for the $\pi_{4}$ mechanism remains larger than $\sigma$ for the $\pi_{5}$ mechanism by about $0.2 \log$ units. Although unlikely, it is possible that the $0.2 \mathrm{log}$ unit difference reflects only experimental variability.

Alpern et al. $(1970 \mathrm{c})$ have previously measured $\sigma$ for the $\pi_{4}$ and $\pi_{5}$ mechanisms, but by the contrastflash technique. In their experiments, the intensity of a contrast-flash $\phi$ (which fell on a steady background $\theta$ ) was adjusted so as to bring a fixcd intensity test flash $\lambda$ to threshold. The test $\lambda$ was a $10 \mathrm{msec}$ flash and the contrast-flash $\phi$, of $100 \mathrm{msec}$ duration, came on $40 \mathrm{msec}$ after the test flash was extinguished. The values of $\sigma$ determined in this manner were about $2 \log$ units higher than those found here. 
One possible reason for the discrepancy is the length of the $\phi$ flash. The semi-saturation constant $\sigma$ is expressed in $\log t \mathrm{tec}$, implying summation over time by the visual system. The $100 \mathrm{msec}$ contrast-flash may be too long to permit the strict summation assumption, suggesting that only the early part of the $\phi$ flash is affecting the threshold. Thus $\phi$, expressed in $\log$ td sec and based on the $100 \mathrm{msec}$ flash, may overestimate $\sigma$.

The duration of $\phi$, while a very likely contributor to the discrepancy, probably cannot account for the full $2 \log$ unit difference. In order to do so, one must assume that only $1 \mathrm{msec}$ of the contrast-flash is effective. A second explanation may lie in the difference between the contrast-flash and increment threshold paradigms. Alpern et al. inferred $\sigma$ by measuring the signal in the surround area necessary to inhibit a test flash. The test flash was distinct from the contrastflash in both space and time. It is not known how the after-flash inhibits the test nor how the inferred estimates of $\sigma$ might be affected by a change in the onset asynchrony of the test and contrast-flashes. ${ }^{2}$

The semi-saturation constant $\sigma$ for the red mechanism also has been explored by King-Smith (personal communication to Alpern, 1974). His observations are increment threshold intensities for a red test flash falling on a flashed white background, with the details of his paradigm varying only slightly from the procedure used here. For $\delta=250 \mathrm{msec}$ and $\delta=1000 \mathrm{msec}$, he found $\sigma$ to be approximately 2.0 $\log t \mathrm{sec}$ with individual subject differences of about $0.2 \log \mathrm{td} \mathrm{sec}$. For $\delta=40 \mathrm{msec}$, King-Smith found a lower value for $\sigma(1.5 \log \mathrm{td}$ sec). However, his method of estimating $\sigma$, different than that used in this paper, is especially sensitive to transient effects (see below) which would indeed cause an underestimate of $\sigma$. Overall, the experiments reported here give a value of $\sigma$ for the $\pi_{5}$ mechanism which agrees quite well with King-Smith's measurements.

\section{Neural transient effects}

lncrement threshold measurements must be affected by (at least) two adaptive mechanisms. The first, reduction of pigment by bleaching, cannot be doubted. It is equally clear that bleaching alone cannot account for all characteristics of adaptation in the cone visual system, and therefore neural mechanisms must be considered. Brief flashed backgrounds were used in the experiments described here in order to explore the neural process alone (except when $\delta=250 \mathrm{msec}$, the flashed background on a single trial always bleached less than $0.2 \%$ and accumulated bleaching was usually zero and never greater

\footnotetext{
' Comparing estimates of $\sigma$ for rods given by the contrast-flash paradigm (Alpern et al., 1970a) and direct increment threshold measurements (Alpern, 1974), the latter procedure provides an estimate of $\sigma$ which is lower by about $0.4 \log$ (scotopic) td sec.

${ }^{3}$ The quantum catch due to a light acting on an unbleached retina $(p=1)$ is proportional to the light's intensity $I$ (expressed in $\mathrm{td}$ ). The quantum catch resulting from the same light intensity falling on a retina with fraction $p<1$ of the pigment unbleached is not precisely proportional to $I p$, but $I p$ does give an excellent approximation which is used in all of the calculations which follow.
}

than $5 \%$; with $d=250 \mathrm{msec}$ non-saturating backgrounds produced bleaches which at most were twice as large).

The transient response of the neural mechanism is not known, but the data in Fig. 3 suggest that the response is positively accelerating with 0 . For low background intensities the increment threshold is about constant over the range of $\dot{j}$. but for litrge values of 0 there appears to bc a strong transient "background on" effect. The cone signal $N$ of equation (1) depends only on the parameters $i$ and $\theta$ land the constants $\sigma$ and $\theta_{D}$ ), neither of which is a function of time, and thus equation (1) takes no account of transience. Therefore, even if equation (1) were generally valid, a transient on response might result in increment threshold measurements with large () and small $\delta$ which rise slightly ahove the curves in Figs. 2 and 3.

\section{Steady backgrounds}

The Alpern et al. (1970c) cone signal equation [equation (1)] is a gain control model in which the background intensity $\theta$ controls the gain [by way of the multiplicative factor $\left.\theta_{D} /\left(0+\theta_{D}\right)\right]$ of the test light channel. Although the semi-saturation constants found here are about 100 times smaller than those found in previous work, the present increment threshold data do fall close to a curve whose general shape is consistent with the cone signal equation. A more remarkable characteristic of equation (1) is that it can account for both the observed cone mechanism saturation with background lights that cause no pigment bleaching (i.e. when brief, flashed backgrounds are used) and the well known absence of saturation with steady backgrounds that reach bleaching equilibrium.

It was shown above that equation (1) implies saturation. Since the quantities $i$ and $\theta$ in (1) are expressed in units of quantum catch, substituting values of $;$ and 0 in units of $t d$ sec and $t d$, respectively, implicitly assumed that no pigment was bleached. However, when steady backgrounds are uscd, as in the classical increment threshold paradigm, pigment bleaching destroys the simple relationship between td (or td $\mathrm{sec}$ ) and quantum catch. If $\dot{\lambda}$ is expressed in td $\sec$ and $\theta$ is given in td, then when fraction of pigment $p$ is unbleached the quantum catches for the test and background fields are reflected by $\lambda p$ and $\theta p$, respectively. ${ }^{3}$ Thus when pigment is bleached by a steady background, the cone signal $N$ is

$$
N=\frac{i p}{i p+\sigma} \frac{\theta_{b}}{\theta p+\theta_{b}} .
$$

At moderate levels of $\theta$ still below the intensity that causes bleaching, $\theta$ is already much larger than $\theta_{D}$. Since $\theta_{p}$ increases with $\theta$, when pigment is bleached equation (1) reduces to

$$
N=\begin{gathered}
\lambda 0_{D} \\
(\lambda p+\sigma) \theta
\end{gathered}
$$

In order to evaluate $N$, the value of $p$ must be determined for each steady background intensity 0 . An expression for $p$ as a function of $\theta$ is provided by the kinetic equation for cone pigment bleaching

$$
-t_{0} \frac{\mathrm{d} p}{\mathrm{~d} t}=\frac{\theta p}{\theta_{0}}-(1-p)
$$


where $t$ is time, $t_{0}$ is the time constant of regeneration (about $120 \mathrm{sec}$ ), $p$ is the fraction of pigment unbleached, $\theta$ is the intensity of the background light, and $\theta_{0}$ is the background level which bleaches $50 \%$ of the pigment at equilibrium (Rushton, 1963; Rushton and Henry, 1968; Alpern, Maaseidvaag and Ohba, 1971).

When the background field is on continuously, an equilibrium condition is reached in which the rate of pigment bleaching exactly equals the rate of pigment regeneration. The net rate of bleaching is zero, so equation $(7)$ is

$$
\begin{aligned}
-t_{0} \frac{\mathrm{d} p}{\mathrm{~d} t} & =\frac{\theta p}{\theta_{0}}-(1-p)=0, \text { which yields } \\
\frac{1}{\theta p} & =\frac{1}{\theta_{0}}+\frac{1}{\theta} .
\end{aligned}
$$

Since $p$ is the fraction of pigment unbleached, $\theta p$ reflects the quantum catch of the background. As $\theta$ increases, $\theta p$ also increases. When the background light $\theta$ is infinitely strong, $\theta p$ attains its maximum value $\theta_{0}$. Thus, the quantum catch upper limit for a steady background light is equal to the quantum catch resulting from the background intensity $\theta_{0}$ when presented to an eye in which all of the pigment is present.

If the test flash intensity $\lambda^{*}$ is the increment threshold for steady background $\theta_{0}$, then substituting into equation (6) gives

$$
N=\frac{2 \lambda^{*} \theta_{D}}{\left(\lambda^{*}+2 \sigma\right) \theta_{0}}
$$

since $\theta_{0}$ bleaches $50 \%$ of the pigment at equilibrium

The increment threshold curve must represent measurements for a constant signal $N$. If we now increase the steady background $\theta$, then the threshold signal $N$ can be reached if and only if there exists a $\lambda$ such that

$$
\frac{\lambda \theta_{D}}{(\lambda p+\sigma) \theta} \geq \frac{2 \lambda^{*} \theta_{D}}{\left(\lambda^{*}+2 \sigma\right) \theta_{0}}=N .
$$

Rearranging and canceling terms yields

$$
\lambda\left(\lambda^{*} \theta_{0}+2 \sigma \theta_{0}-2 \lambda^{*} p \theta\right) \geq 2 \lambda^{*} \sigma \theta .
$$

All of the variables and constants in inequality (11) are positive. In order that saturation does not occur with any steady background, for every value of $\theta$ there must correspond a value of $\lambda$ such that the inequality is satisfied. The term on the right side of (11) is positive; thus when the term

$$
\left(\lambda^{*} \theta_{0}+2 \sigma \theta_{0}-2 \lambda^{*} p \theta\right)
$$

is negative the inequality will be satisfied only if $\lambda$ is negative. Of course $\lambda$ can never be negative and therefore saturation will occur when the term (12) is less than zero. On the other hand, no saturation with steady backgrounds can occur if

$$
\lambda^{*} \theta_{0}+2 \sigma \theta_{0}-2 \lambda^{*}(\theta p)>0
$$

${ }^{4}$ I am grateful to Dr. Mathew Alpern for performing the densitometry. for every $\theta$. Since $\theta p$ increases with $\theta$ to its upper limit $\theta_{0}$, the left-hand side of (13) will be smallest when $\theta_{0}$ is substituted for $(\theta p)$. Making this substitution gives

$$
\lambda^{*} \theta_{0}+2 \sigma \theta_{0}-2 \lambda^{*} \theta_{0}>0
$$

which, upon canceling and collecting terms, yields

$$
\lambda^{*}<2 \sigma .
$$

Thus no saturation with steady backgrounds can occur if $\lambda^{*}$, the increment threshold for steady background $\theta_{0}$, is less than $2 \sigma$.

Of course, it is well known that steady backgrounds do not saturate the cone visual system. Inequality (14) is a theoretical prediction based on equation (1), and may be tested experimentally. First, the intensity $\theta_{0}$ must be determined. Using the retinal densitometer, the value of $\theta_{0}$ (the intensity which bleaches half the pigment at equilibrium) was determined for the subject whose increment threshold measurements are reported above. ${ }^{4}$ Physical constraints of the apparatus permitted densitometry on the left eye only; it is assumed that $\theta_{0}$ for the subject's right eye (which was used in the increment threshold experiments) is the same. Measurements made with a red measuring light $(\lambda=631.5 \mathrm{~nm})$ are shown in Fig. 4 which indicates $\theta_{0}=4.4 \log$ td for the red cone pigment. Rushton (1958) showed that the red and green cone pigments regenerate at the same rate and that a white (tungsten) light bleaches both pigments at an equal rate. Thus $0_{0}=4.4 \log$ td can be approximated for the green cone pigment also.

Given $\theta_{0}$, it is a simple matter to measure the increment threshold $\lambda^{*}$; the data are presented in Figs. 2 and 3. Error bars ( \pm 1 S.E. mean) are shown for the measurements of $\lambda^{*}$. For the $\pi_{5}$ mechanism, $\lambda^{*}=1.95 \pm 0.02$ (S.E. mean) $\log \mathrm{td} \sec$ and $2 \sigma=2.3$ $\log$ td sec. For the $\pi_{4}$ mechanism, $\lambda^{*}=2.07 \pm 0.06$ (S.E. mean) $\log$ td sec and $2 \sigma=2.75 \log$ td sec. There can be little doubt that inequality (14) is satisfied.

Returning to inequality (11), the threshold value of $\lambda$ is

$$
\lambda=\frac{2 \lambda^{*} \sigma \theta}{\lambda^{*} \theta_{0}+2 \sigma \theta_{0}-2 \lambda^{*} p \theta}
$$

since term (12) is positive. Substituting for $p$ gives

$$
\begin{aligned}
\lambda & =\frac{2 \lambda^{*} \sigma \theta}{\lambda^{*} \theta_{0}+2 \sigma \theta_{0}-\frac{2 \lambda^{*} \theta \theta_{0}}{\theta+\theta_{0}}} \\
& =\frac{2 \lambda^{*} \sigma}{\theta_{0}\left(2 \sigma-\lambda^{*}\right)} \frac{\theta\left(0+\theta_{0}\right)}{\theta+\theta_{0}\left(\frac{2 \sigma+\lambda^{*}}{2 \sigma-\lambda^{*}}\right)} .
\end{aligned}
$$

Taking logarithms,

$$
\log \lambda=\log \theta+\log \left(\theta+\theta_{0}\right)-\log \left(\theta+K \theta_{0}\right)+c,
$$

where constants

$$
K=\frac{2 \sigma+\lambda^{*}}{2 \sigma-\lambda^{*}}
$$




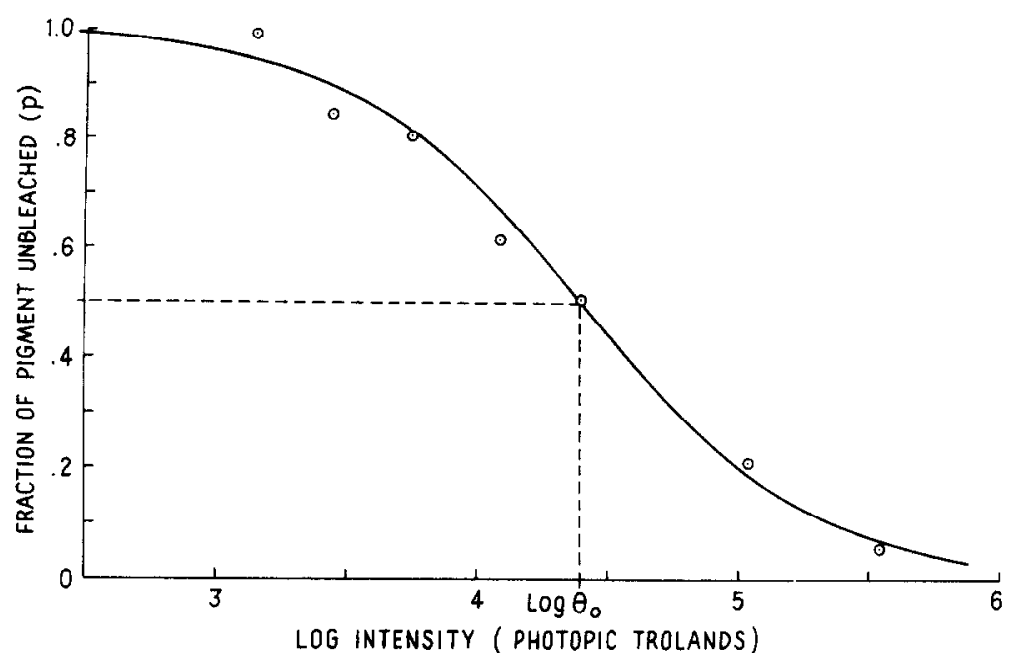

Fig. 4. Fraction of pigment unbleached at equilibrium as a function of white light intensity. The pigment measured is erythrolabe (wavelength of measuring light $631.5 \mathrm{~nm}$ ).

and

$$
c=\log \frac{2 \lambda^{*} \sigma}{\theta_{0}\left(2 \sigma-\lambda^{*}\right)}
$$

As $\theta$ becomes large, equation (15) gives the WeberFechner relationship. Substituting the observed values for $\sigma$ and $\lambda^{*}$ roughly estimates $K=2.9$ for the $\pi_{5}$ mechanism and $K=1.5$ for the $\pi_{4}$ mechanism. These estimates are not precise, but do suggest that $K$ is of order $10^{\circ}$.

Regardless of the general validity of equation (1), the preceding analysis demonstrates that a very simple gain control model is capable of accounting for receptor mechanism saturation in the absence of bleaching, and non-saturating, Weber's law type behavior when steady backgrounds are used. The absence of saturation results from the pigment bleaching caused by steady backgrounds; bleaching can inhibit or eliminate saturation because the pigment depletion not only reduces the quantum catch of the incremental stimulus but also reduces the quantum catch of the adapting light, thus reducing the attenuation due to the gain control.

Acknowledgements-This work was supported by a National Science Foundation Graduate Fellowship to the author, and National Institutes of Health Grants EY-00197-17 to Dr. Mathew Alpern and GM-01231-11 to The University of Michigan. I am indebted to Dr. Alpern for his generous substantive contributions to this research.

\section{REFERENCES}

Aguilar M. and Stiles W. S. (1954) Saturation of the rod mechanism of the retina at high levels of stimulation. Optica Acta 1, 59-65.

Alpern M. (1965) Rod-cone independence in the after-flash effect. J. Physiol., Lond. 176, 462-472.

Alpern M. (1974) What is it that confines in a world without color? Investve Ophth. 13, 648-674.
Alpern M.. Maaseidvaag F. and Ohba N. (1971) The kinetics of cone visual pigments in man. Vision Res. 11, $539-549$.

Alpern M. and Rushton W. A. H. (1965) The specificity of the cone interaction in the after-flash effect. $J . P h y$ siol., Lond. 176, 473-482.

Alpern M. and Rushton W. A. H. (1967) The nature of the rise in threshold produced by contrast-flashes. $J$. Physiol., Lond. 189, 519-534.

Alpern M., Rushton W. A. II. and Torii S. (1970a) The size of rod signals. J. Physiol., Lond. 206, 193-208.

Alpern M. Rushton W. A. H. and Torii S. (1970b) The attenuation of rod signals by backgrounds. J. Physiol. Lond. 206, 209-227.

Alpern M., Rushton W. A. H. and Torii S. (1970c) Signals from cones. J. Physiol., Lond. 207, 463.475.

Geisler W. S. (1975) Visual adaptation and inhibition. $\mathrm{Ph} . \mathrm{D}$. dissertation, Indiana University.

King-Smith P. E. (1975) Visual detection analysed in terms of luminance and chromatic signals. Nature, Lond. 255, 69-70.

King-Smith P. E. and Webb J. R. (1974) The use of photopic saturation in determining the fundamental spectral sensitivity curves. Vision Res. 14, 421-429.

Rushton W. A. H. (1958) Kinetics of cone pigments measured objectively on the living human fovea. Ann. N.Y. Acad. Sci. 74 (Art 2), 291--304.

Rushton W. A. H. (1963) Cone pigment kinetics in the protanope. J. Physiol., Lond. 168, 374-388.

Rushton W. A. H. and Henry G. H. (1968) Bleaching and regeneration of cone pigments in man. Vision Res. 8 , $617-631$.

Stiles W. S. (1939) The directional sensitivity of the retina and the spectral sensitivities of rods and cones. Proc. R. Soc. B, 127, 64-105.

Stiles W. S. (1949) Increment thresholds and the mechanisms of color vision. Documenta ophth. 3, 138-163.

Stiles W. S. (1959) Color vision: the approach through increment-threshold sensitivity. Proc. natn. Acad. Sci. Wash. 45, 100-114.

Westheimer G. (1966) The Maxwellian view. Vision Res 6, 669-682.

Wyszecki G. and Stiles W. S. (1967) Color Science: Concepts and Methods, Quantitative Data and Formulas. Wiley, New York. 